\title{
Anatomía Topográfica del Nervio Laríngeo Superior: Importancia Quirúrgica en las Tiroidectomías
}

\author{
Topographic Anatomy of the Upper Laryngeal Nerve: Surgical Importance in Thyroidectomies
}

\author{
Blás Antonio Medina-Ruíz; Joaquín Julián Medina-Izcurdia²; Blás Agustín Medina-Izcurdia; \\ Rocío Belén Martínez-Vera ${ }^{3}$; Pedro Ramón Martínez-Vera ${ }^{3}$; Clara Elba Izcurdia ${ }^{4}$ \& Nicolás Ernesto Ottone ${ }^{5,6,7}$
}

MEDINA-RUIZ, B. A.; MEDINA-IZCURDIA, J. J.; MEDINA-IZCURDIA, B. A.; MARTÍNEZ-VERA, R. B.; MARTÍNEZVERA, P. R.; IZCURDIA, C. E. \& OTTONE, N. E. Anatomía topográfica del nervio laríngeo superior: Importancia quirúrgica en las tiroidectomías. Int. J. Morphol., 38(4):766-773, 2020.

RESUMEN: En las cirugías sobre la glándula tiroides se ha prestado mucha atención al manejo del nervio laríngeo inferior y de las glándulas paratiroides, no así del ramo lateral del nervio laríngeo superior, el cual es satélite del pedículo vascular superior de la glándula tiroides. El manejo del polo superior de la glándula tiroides requiere de un conocimiento acabado de su anatomía topográfica del área y está determinada por sus relaciones más importantes, dadas por el citado nervio y la arteria tiroidea superior principalmente. En este trabajo se pretende estudiar estas relaciones en base a la disección meticulosa del triángulo laringo-esternotiroideo ("Triángulo de Joll") de 25 cadáveres adultos formolizados. Como hallazgo relevante se informa que los "nervios en riesgo", según la clasificación de Cernea, que se basa en una distancia menor a un centímetro en el entrecruzamiento del nervio con la arteria tiroidea superior con respecto al polo superior de la glándula tiroides, es del $52 \%$ para el lado derecho y $44 \%$ para el lado izquierdo del cuello. El origen bajo de la arteria a nivel de la bifurcación carotídea se presenta asociada a un mayor número de "nervios en riesgo" en el lado izquierdo. Según el punto de penetración del ramo lateral del nervio laríngeo superior en el músculo constrictor inferior de la faringe se establece la clasificación de Friedman, muy útil sobre todo en cirugías ayudadas por la neuroestimulación. En esta clasifiacción los "nervios en riesgo" son aquellos que transcurrren superficial al músculo, mientras que los "nervios protegidos" serían aquellos que perforan el músculo en su porción superior. En este trabajo los "nervios en riesgo" se presentaron del lado izquierdo en el $56 \%$ de los casos y del derecho en el $60 \%$, mientras que los "nervios protegidos" en el $24 \%$ y $16 \%$, respectivamente.

PALABRAS CLAVE: Ramo lateral del nervio laríngeo superior; Arteria tiroidea superior; Polo superior de la glándula tiroides; Anatomía topográfica.

\section{INTRODUCCIÓN}

La gran mayoría de los artículos relacionados a la cirugía tiroidea hacen mención al manejo correcto del nervio laríngeo inferior y de las glándulas paratiroides, para evitar sus lesiones y respectivas secuelas, las cuales son muy manifiestas y con una marcada disminución de la calidad de vida en aquellos pacientes que las sufren. Sin embargo no merece tal atención el ramo lateral del nervio laríngeo superior (RLNLS), íntimamente relacionado al polo superior de la glándula tiroides y satélite de su pedículo vascular superior. Tal situación lleva a considerar al citado como "el ner- vio descuidado en la cirugía tiroidea" (Ekhar et al., 2018). El nervio laríngeo superior (NLS) se origina a nivel de la parte inferointerna del ganglio plexiforme del nervio vago, continúa un trayecto descendente dirigiéndose hacia la faringe dividiéndose en dos ramos, el ramo superior, el ramo interno (RINLS), que continúa a su nervio de origen siguiendo el asta mayor del hueso hiodes transcurriendo entre el músculo tirohioideo y la membrana tirohiodea, perforando esta última estructura, en compañía de los vasos laríngeos superiores, para terminar en el espesor de los repliegues

\footnotetext{
'Profesor Adjunto de Anatomía descriptiva y Topográfica. Facultad de Ciencias Médicas- Universidad Nacional de Asunción (FCM-UNA), Paraguay. ${ }^{2}$ Estudiante Universitario. Carrera de Medicina. Universidad de Integración de las Américas (UNIDA), Paraguay. ${ }^{3}$ Estudiante Universitario. Carrera de Medicina. FCM-UNA, Paraguay.

${ }^{4}$ Podóloga Universitaria. FCM-UNA, Paraguay.

${ }^{5}$ Laboratorio de Plastinación y Técnicas Anatómicas, Centro de Investigación en Ciencias Odontológicas (CICO), Facultad de Odontología, Universidad de La Frontera, Temuco, Chile.

${ }^{6}$ Centro de Excelencia en Estudios Morfológicos y Quirúrgicos (CEMyQ), Universidad de La Frontera, Temuco, Chile. ${ }^{7}$ Programa de Doctorado en Ciencias Morfológicas, Universidad de La Frontera, Temuco, Chile.
} 
aritenoepliglóticos donde da sus filetes terminales, todos sensitivos destinados a ambas caras del cartílago epliplótico, valéculas y base de lengua a través de sus filetes anteriores. Sus filetes medios y posteriores inervan a los repliegues aritenoepiglóticos y la región subglótica, así como la cara posterior de la laringe y a la hipofaringe. Uno de estos filetes desciende verticalmente entre el cricoaritenoideo posterior y la mucosa para anastomosarse con un filete ascendente, también sensitivo del nervio laríngeo inferior (NLI), conocido como asa nerviosa de Galeno (Testut \& Latarjet, 1984).

El ramo inferior o lateral del nervio laríngeo superior (RLNLS), de menor calibre con respecto al ramo inerno, nace a la altura del hueso hiodes, se dirige oblicuamente hacia abajo, posterior al músculo esternotiroideo, relacionándose a este nivel con a la Arteria tiroidea superior (ATS), que corre en un pano más profundo al mismo. Desciende a lo largo de la inserción anterior en del músculo constrictor inferior de la faringe, entre el músculo y la glándula tiroides, recorriendo su cara superficial y perforándolo a una altura variable e inervándolo, rodea al asta inferior del cartílago tiroideo para llegar al músculo cricotiroideo al que también inerva, para terminar en la mucosa subglótica tras perforar la membrana cricotirodea (Estrela et al., 2011).

El RLNLS es principalmente motor, pero termina como ramo sensitivo de la mucosa subglótica a nivel de la membrana cricotiroidea, recibiendo además anastomosis del simpático cervical, específicamente provenientes del ganglio cervical superior, asi como anastomosis motoras provenientes del NLI, que colaborarían en la inervación motora de otros múscculos intrínsecos de la laringe (Sanders et al., 1993; Wu et al., 1994; Maranillo et al., 2003; Estrela et al.; Gurleyik, 2018).

La relación del RLNLS con el polo superior y el pedículo vascular superior de la glándula tiroides, especialmente la ATS, se da en el triángulo laringo-esternotiroideo ("Triángulo de Joll") y es relevante, a tal punto que la relación recíproca entre estos elementos anatómicos sirvió como base a la clasificación de Cernea, la cual es muy utilizada hasta la fecha para el estudio del nervio y su manejo seguro durante la cirugía tiroidea, específicamente en el tiempo de la ligadura y sección de los vasos tiroideos superiores. Esta clasificación presenta tres tipos, en el tipo 1 el entrecruzamiento entre el RLNLS y la ATS se da por encima de $1 \mathrm{~cm}$ del polo superior de la glándula tiroides. En el tipo 2 este entrecruzamiento se da por debajo del centímetro, subdividiendose en 2a por encima de la glándula tiroides y $2 b$ por debajo del plano que pasa a nivel del margen superior del polo. Estos últimos son considerados de " alto riesgo"(Cernea et al., 1992).
Kierner et al. (1998) presentaron una modificación a la clasificación de Cernea, con cuatro tipos. El tipo 1 se corresponde al tipo 1 de Cernea, y el tipo 2 de Kierner al tipo 2a de Cernea, así como el tipo 3 al 2 b y le agrega el tipo 4 en el que el RLNLS transcurre entre las ramas terminales de la ATS.

Friedman et al. (2002) establecieron una clasificación complementaria a la de Cernea basándose en el sitio de perforación del músculo constrictor inferior de la faringe por parte del RLNLS, así determinaron que aquellos nervios que corren paralelo a la ATS, superficial al músculo constrictor inferior de la faringe hasta el MCT pertenecen al gruppo 1. Los del grupo 2 perforan al músculo constrictor inferior de la faringe en su tercio inferior y los del grupo 3 en su tercio superior. Los del grupo 1 corresponden a los "nervios en riesgo" (Friedman et al.).

Algunos autores de centros muy especializados en cirugía tiroidea sugieren incluso, en base a esta relación, evitar el uso de electrocauterio en el manejo del polo superior de la glándula tiroides disminuir el riesgo de lesión nerviosa. El detalle técnico de buscar el nervio durate la cirugía en base a estas relaciones anatómicas los hace prescindir de utilizar de modo sitemático el neuroestimulador para tal efecto (Pagedar \& Freeman, 2009; Ekhar et al.).

Es de destacar que gran cantidad de centros asistenciales, con basta experiencia en cirugía tiroidea, usan rutinariamente el neuroestimulador para evitar lesionar el nervio durante el acto operatorio, considerando este método más seguro que la búsqueda por disección y visualización directa (Wang et al., 2017).

La acción principal del RLNLS es la inervación motora del músculo cricotiroideo, el cual tensa las cuerdas vocales produciendo sonidos de alta frecuencia, mayores a 150 $\mathrm{Hz}$, manteniendo a su vez en tensión a las mismas durante la fonación, permitiendo así una fonación de tonos altos. Además optimiza la respiración evitando la broncoaspiración durante la deglución (Estrela et al.; Dessie, 2018).

La lesión quirúrgica del RLNLS se reporta con una prevalencia del 0 al $58 \%$, y se manifiesta en muchos casos con una sintomatología menos florida que la relacionada con las lesiones del nervio laríngeo inferior y de las glándulas paratiroides, lo cual probemente esté relacionada con la menor atención brindad a su cuidado por parte de algunos equipos quirúrgicos que se dedican a la patología tiroidea(Ekhar et al.).

Sin embargo para algunas personas que utilizan la voz como recurso los periodistas, docentes, cantantes, sobre todo mujeres, su lesión puede resultar catastrófica, tanto es así que 
se llegó a denominar a esta estructura como "nervio de GalliCurci”, debido a la lesión del nervio que sufriera la catante de ópera Amelita Galli Curci tras una tiroidectomía con anestesia local y que truncara su carrera artística, aunque algunos autores sostienen que el cambio de voz que sufriera probablemente estaba relacionado con su hipotiroidismo secundario (Whitfield et al., 2010; Bruno, 2019).

En este tratajo se detalla la relación que guardan entre sí el RLNLS con la ATS y el polo superior de la glándula tiroides (PSGT) en 30 cadáveres humanos adultos formolizados.

El objetivo general del estudio consistió en establecer la relación entre el ramo lateral del nervio laríngeo superior, la arteria tiroidea superior y el polo superior de la glándula tiroides. A su vez, entre los objetivos específicos, buscamos:

-Determinar el porcentaje de "nervios en riesgo" según la altura en $\mathrm{cm}$ del entrecruzamiento entre la arteria tiroidea superior y el ramo lateral del nervio laríngeo superior con respecto al polo superior de la glándula tiroides.

- Evaluar la asociación entre el nivel de entrecruzamiento del RLNLS y la ATS según el sitio de origen de la arteria. - Considerar el punto de perforación del RLNLS al músculo constrictor inferior de la faringe.

- Determinar la posible asociación entre el sitio de perforación del músculo constrictor inferior de la faringe por parte del RLNLS y el entrecruzamiento del mismo con la ATS.

\section{MATERIAL Y MÉTODO}

Estudio descriptivo de corte transversal basado en la disección cadavérica de 25 cadáveres humanos adultos formolizados al $10 \%, 21$ del sexo masculino y 4 del sexo femenino, realizados entre los meses de marzo de 2010 a diciembre de 2019.

Para la disección cadavérica e utilizó instrumental quirúrgico perteneciente a la Cátedra de Anatomía Descriptiva y Topográfica de la Facultad de Ciencias Médicas, de la Universidad Nacional de Asunción, Paraguay. Las mediciones se realizaron con una regla centimetrada. Las imágenes fueron obtenidas con una cámara digital Nikon Coolpix L 340.

Los datos fueron cargados en una planilla Excel, codificados y analizados con el programa Epi-Info 2007 (CDC) con estadística descriptiva y para evaluar probables asociaciones entre estas, estudiadas mediante tablas de contingencia y las pruebas de Chi cuadrado. Las asociaciones se consideran significativas para $\mathrm{p}<0,05$.
Criterios de inclusión:Cadáveres humanos adultos, de ambos sexos, formolizados y disecados con la misma sistematización por el investigador principal.

Criterios de exclusión: Cadáveres con cirugías cervicales previas, con tumores cervicales o bocios o cuerpos en malas condiciones de conservación para la disección cervical detallada y meticulosa.

El trabajo siguió todas las normas éticas contenidas en la Declaración de Helsinki, de la Organización Mundial de la Salud, para investigación biomédica, dando un trato ético y respetuoso a los cadáveres utilizados. Se garantizó la confidencialidad de los datos, y la integridad y la dignidad de las personas fallecidas (Manzini, 2000).

\section{RESULTADOS}

Fueron disecados 25 cadáveres humanos adultos formolizados, en buen estado de conservación, 21 del sexo masculino $(84 \%)$ y 4 del sexo femenino $(16 \%)$.

Se realizó una incisión vertical medial submentoniana-supraesternal, con amplianes laterales submandibulares y supraclaviculares bilaterales. Se levantaron colgajos a nivel subplatismal. Se realizó apertura de la línea media infrahioidea, rebatiéndose lateralmente el músculo esternohiodeo. Se seccionó parcialmente el músculo esternotiroideo para facilitar su separación del polo superior de la glándula tiroides. En todos los casos se disecó el RLNLS y la ATS en todo su trayecto desde sus orígenes. Se determinó la distancia entre el margen superior del polo superior de la glándula tiroides y el punto de cruzamiento entre el RLNLS y la ATS.
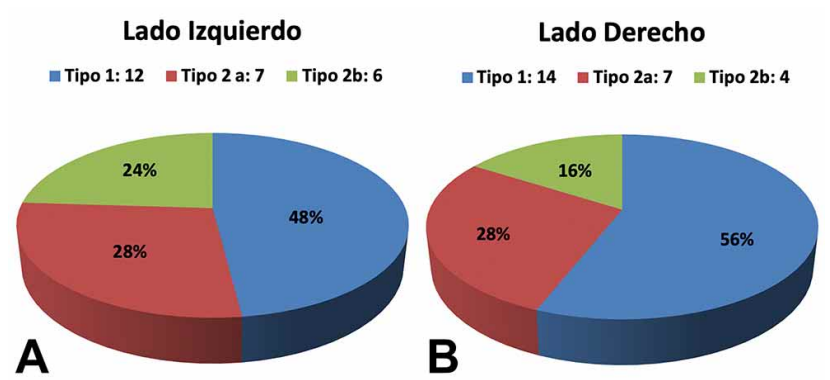

Fig. 1. A. Presentación de los tipos de entrecruzamiento RLNLS/ ATS (Ramo lateral del nervio laríngeo superior / Arteria tiroidea superior) del lado izquierdo en relación al polo superior de la glándula tiroides (según la Clasificación de Cernea). B. Presentación de los tipos de entrecruzamiento RLNLS/ATS del lado derecho en relación al PSGT (según la Clasificación de Cernea). 
Este entrecruzamiento se produjo a una distancia menor a $1 \mathrm{~cm}$ del PSGT en el $52 \%$ de los casos en el lado izquierdo y en el $44 \%$ en el lado derecho. La Figura 1 muestra la presentación de los tipos de Cernea en los lados izquierdo y derecho. La simetría en los tipos de Cernea se presentó en el $64 \%$ (16 casos).

En la Figura 2A-B se observa el entrecruzamiento del RLNLS con la ATS a una distancia mayor al centímetro por encima del PSGT
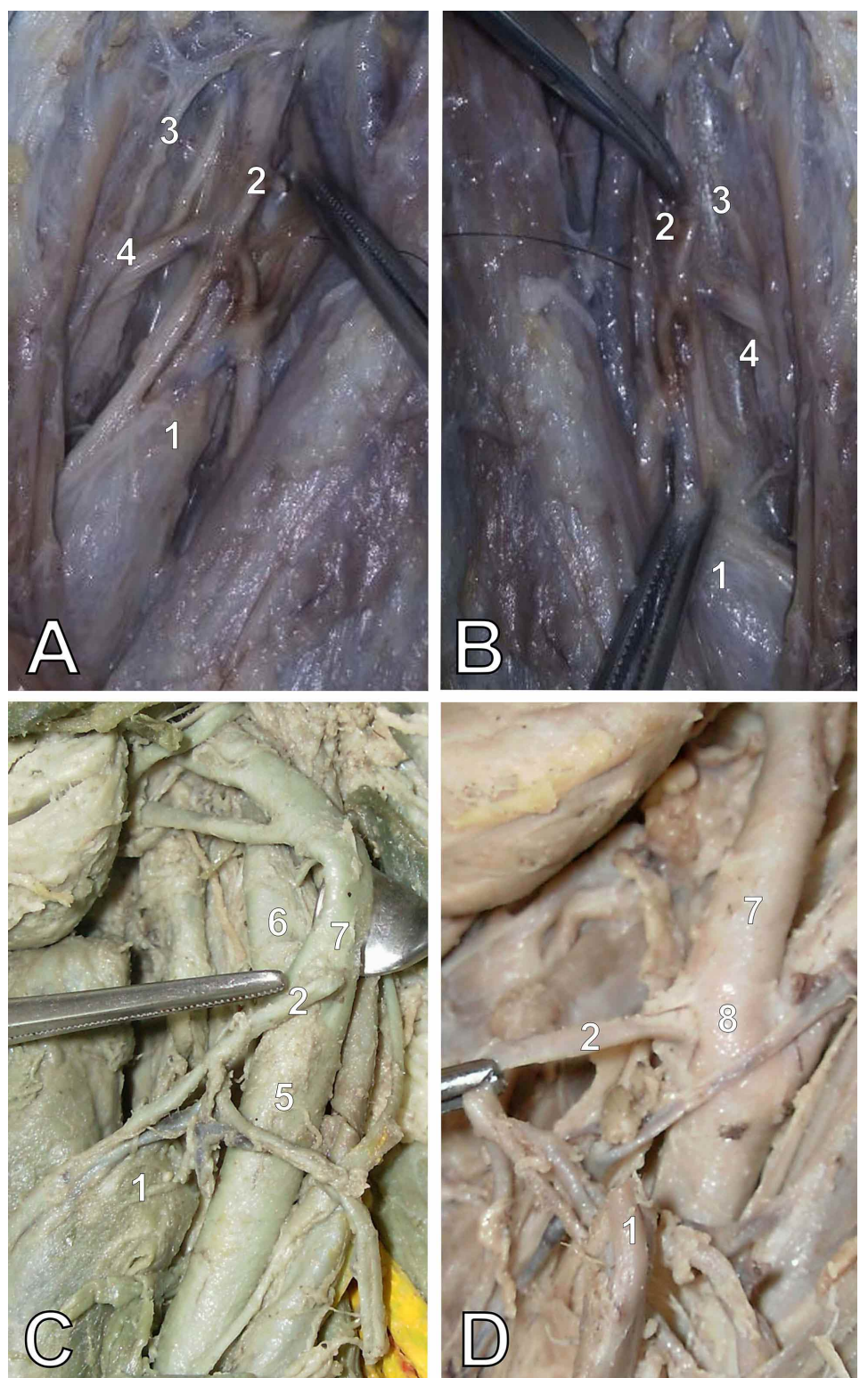

Fig. 2. En las figuras A (lado izquierdo) y B (lado derecho), correspondientes al mismo cadáver, se identifica la simetría en los tipos de Cernea. C. Arteria tiroidea superior que nace de la Arteria carótida externa. D. Arteria tiroidea superior que nace a nivel de la bifurcación carotídea en el lado izquierdo. 1, Polo superior de la glándula tiroides; 2, Arteria tiroidea superior; 3, Rama lateral del nervio laríngeo superior; 4, Músculo constrictor inferior de la faringe, 5 , Arteria carótida común; 6 . Arteria carótida interna; 7, Arteria carótida externa; 8, bifurcación carotídea.
(Tipo 1 de la Clasificación de Cernea). En el $64 \%$ de los casos la ATS nació de la ACE, mientras que este origen se presentó a nivel de la bifurcación carotídea en el $36 \%$ (9 casos) en ambos lados por igual, sin embargo hubo simetría solo en 18 casos $(72 \%)$.

En la Figura 2C se muestra el origen de la ATS en la ACE, mientras que en las Figuras 2D y $3 \mathrm{~A}$, se ven dos arterias originadas en la bifurcación carotídea.

Con respecto al sitio de penetración del RLNLS en el músculo constrictor inferior de la faringe encontramos en el lado izquierdo que el $24 \%$ lo hizo a nivel de la parte superior del mismo, y el $20 \%$ en la parte inferior (Fig. 3B), mientras que el $56 \%$ restante trascurrió superficial al músculo hasta su proximidad con el MCT. Esta última situación se presentó en el $60 \%$ en el lado derecho, mientras que la perforación alta del músculo se observó en el $16 \%$. La simetría en cuanto a la presentación de los tipos de Friedman se observó en 14 casos (56 \%). En la Tabla I se observa la presentación de los tipos de Cernea y de Friedman según el lado del cuello estudiado.

En las Figuras 3C-D pueden observarse el paralelismo entre la ATS y el RLNLS. En la Figura $3 \mathrm{E}$ se muestra esta ausencia de paraleleismo entre los tipos 1 de Cernea y 3 de Friedman. No hubo asociación entre las variables sexo del cadáver y la presentación de los tipos de Cernea y de Friedman, así como tampoco con el origen de la ATS y la clasificación de Friedman. Sin embargo si hubo asociación positiva, de significación estadística entre el nivel de origen de la ATS en el tronco carotideo y el nivel de entrecruzamiento de esta arteria con el RLNLS respecto al PSGT (Clasificación de Cernea) en el lado izquerdo (OR: 17,6; IC $95 \%$ : 1,7-181.2; p: 0,005).

Asi mismo no hubo asociación entre la simetríade los tipos de Cernea y Friedman, sin embargo si se observó asociación positiva entre la simetríade los tipos de Cernea y la simetríaen el origen de la ATS del lado izquierdo, pero sin significación estadística (OR: 8,7; IC 95 \%: 1,2-63,4; p: 0,02).

La Tabla I muestra un resumen de los hallazgos en este trabajo de manera comparativa en ambos lados del cuello. 

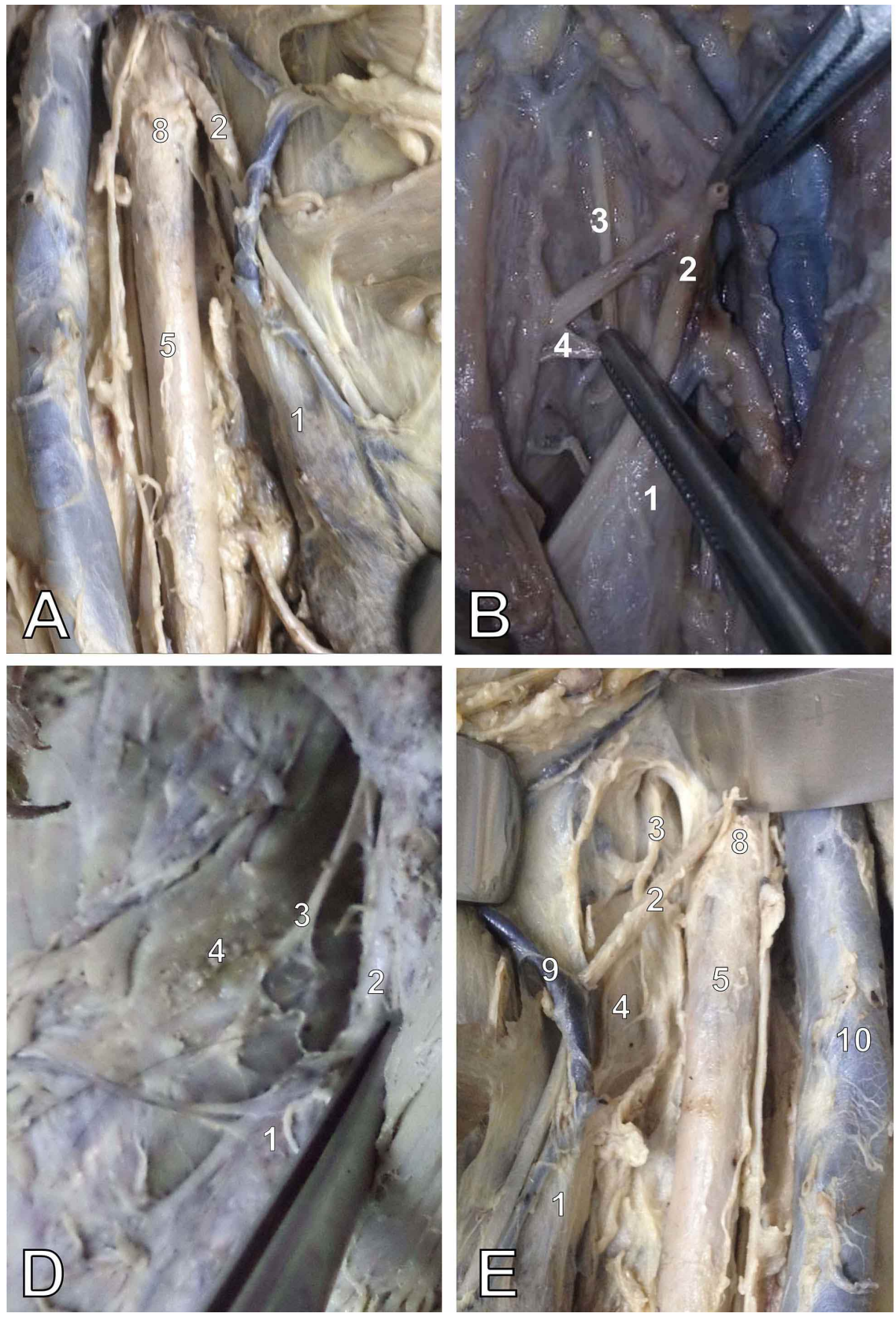

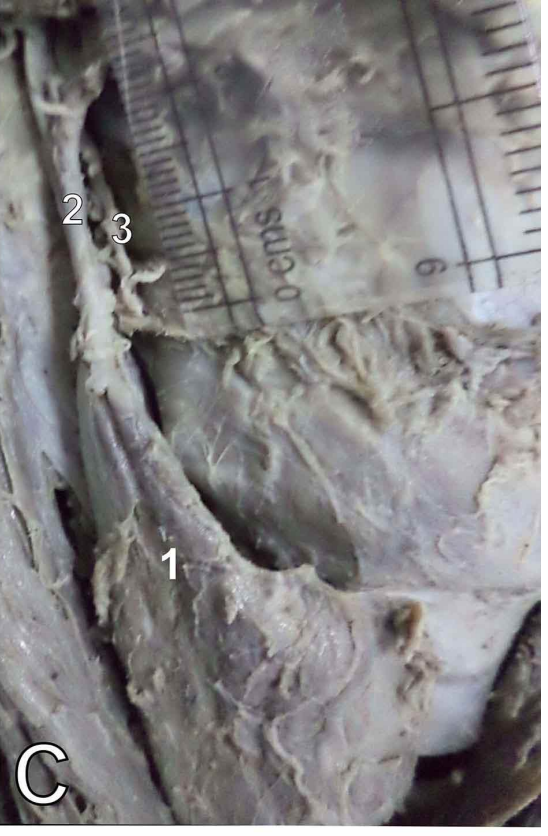

Fig. 3. A. Origen de la A. tiroidea superior a nivel de la bifurcación carotídea en el lado derecho. B. Especimen lado izquierdo, con presencia del Tipo 2 de Friedman: el RLNLS perfora el tercio inferior del M. constrictor inferior de la faringe. El entrecruzamiento neurovascular se da $1 \mathrm{~cm}$ sobre el polo superior de la glándula tiroides. C. Especimen lado derecho, con presencia de Cenea tipo 1; Friedman tipo 1. D. Especimen lado izquierdo con tipo Cernea 2a; Friedman 2. E. Especimen con Cernea tipo 1; Friedman tipo 3 , lado izquierdo. Se nota la ausencia del paralelismo entre el RLNLS y la A. tiroidea superior debido a que el nervio perfora al $\mathrm{M}$. constrictor inferior en su porción superior. 1 , Polo superior de la glándula tiroides; 2, A. tiroidea superior; 3, Rama lateral del N. laríngeo superior; 4, M. constrictor inferior de la faringe, 5, A. carótida común; 6 . A. carótida interna; 7, A. carótida externa; 8, bifurcación carotídea; 9, V. tiroidea superior; 10, V. yugular interna.

Tabla I. Relaciones entre el RLNLS con la ATS y con el músculo constrictor inferior de la faringe.

\begin{tabular}{|c|c|c|c|c|c|}
\hline \multicolumn{6}{|c|}{ Tipos de Cernea } \\
\hline & Lado Izquierdo & & & Lado Derecho & \\
\hline Tipo 1 & Tipo 2a & Tipo 2b & Tipo 1 & Tipo 2a & Tipo 2b \\
\hline $12(48 \%)$ & $7(28 \%)$ & $6(24 \%)$ & $14(56 \%)$ & $7(28 \%)$ & $4(16 \%)$ \\
\hline \multicolumn{6}{|c|}{ Tipos de Friedman } \\
\hline & Lado Izquierdo & & & Lado Derecho & \\
\hline $\begin{array}{c}\text { Tipo 1 } \\
14(56 \%)\end{array}$ & $\begin{array}{c}\text { Tipo 2 } \\
5(20 \%)\end{array}$ & $\begin{array}{c}\text { Tipo 3 } \\
6(24 \%)\end{array}$ & $\begin{array}{c}\text { Tipo 1 } \\
15(60 \%)\end{array}$ & $\begin{array}{c}\text { Tipo 2 } \\
6(24 \%)\end{array}$ & $\begin{array}{c}\text { Tipo 3 } \\
4(16 \%)\end{array}$ \\
\hline
\end{tabular}




\section{DISCUSIÓN}

La lesión del RLNLS es frecuente y está esta relacionada básicamente con que muchos cirujanos prefieren evitarlo, sin identificarlo ni exponerlo, incumpliendo el concepto básico en cirugía de cabeza y cuello de exponer y preservar las estructuras nobles (Aina \& Hisham, 2001).

El porcentaje de reconocimiento del nervio durante el acto operatorio es reportado entre el 82 al $98,5 \%$, ya sea con la utilización o no del neuromonitoreo intraquirúrgico. No todos los centros asistenciales disponen del neuroestimulador para individualizar el nervio, de todos modos e independientemente de este detalle, el conocimiento de la anatomía topográfica del RLNLS es vital para su reconocimiento. El uso del neuroestimulador logra un mayor individualización del nervio y se nutre mejor del aporte brindado por la clasificación de Friedman (Aina \& Hisham; Friedman et al.; Kandil et al., 2015; Arun et al., 2016; Ekhar et al.; Bruno; Gurleyik et al., 2019).

Antes de la clasificacion de Cernea, Mosman y De Weese consideraron como "nervios de alto riesgo" a aquellos en los que el entrecruzamiento con la ATS se producía a menos de $1 \mathrm{~cm}$ del PSGT en un artículo publicado en "Surgery, Gynecology \& Obstetrics" en el año 1968 (Cernea et al.).

La estatura es un detalle que puede influir en la distancia existente entre el entrecruzamiento vasculonervioso estudiado y el PSGT. Whitfield et al. encontraron un mayor número de nervios en riesgo, es decir a menos de $1 \mathrm{~cm}$ del PSGT. En este trabajo no se consideró esta variable.

La edad también influiría en las relaciones anatómicas estudiadas debido al aumento de la grasa corporal, la reducción de los tejidos óseos y muscular, la calcificación de los cartílagos laríngeos y algunos otros cambios (Estrela et al.). Tampoco fue considerado este detalle, sin embargo todos nuestros cadávres fueron de adultos mayores.

El tamaño de la glándula tiroides es otro detalle importante, pues el aumento de su tamaño modifica de manera notoria la relación del PSGT con respecto a la ATS, el RLNLS y el entrecruzamiento en ambas estructuras. Es asi como en los grandes bacios este entrecruzamiento está mucho más proximo al PSGT (Aina \& Hisham; Pagedar \& Freeman; Estrela et al.; Ekhar et al.).

Esta relación entre el aumento de tamaño de la glándula tiroides y su relación con las estructuras adyavcnetes puede predecirse en el preoperatorio puesto que una glán- dula tiroides en que la ecografía muestre un volumen mayor a $50 \mathrm{cc}$ presenta un mayor número de nervios en riesgo. Menon et al. (2017) encontraron que bocios mayores a 50 cc presentaron mayor número de entrecruzamientos vasculonerviosos a una distancia menor al $\mathrm{cm}$ del PSGT (Ha et al., 2015; Menon et al.).

Mizrachi et al. (2015), en 35 años de actividad quirúrgica en uno de los mayores centros quirúrgicos a nivel mundial, presentan una propuesta de clasificación anatómica del PSGT que tiene implicancia en la relación de la glándula tiroides con el RLNLS, la ATS y el entrecruzamiento entre ambos. El tipo 1 propuesto es aquel en que el PSGT tiene una forma piramidal y en el que los elementos del pedículo superior se encuentran próximos entre si. En el tipo 2 el PSGT es más ancho y los elementos del pedículo se encuentran un poco más dispersos, mientras que en el tipo 3 el polo es muy ancho con los elementos del pedículo muy dispersos, lo cual conlleva un mayor riesgo de sangrado y lesion nerviosa durante el acto quirúrgico (Mizrachi et al.). Este detalle tampoco fue estudiado en este trabajo.

Se consideran "nervios en riesgo" a aquellos en los que su entrecruzamiento con la ATS se da a una distancia menor a $1 \mathrm{~cm}$, ya sea por encima, a nivel o por debajo del PSGT. Esto es tipo 2, tanto a como b, de la clasificación de Cernea, así como los tipos 2, 3 y 4 de Kierner (Cernea et al.; Kierner et al.). En este trabajo no encontramos ningún caso que pueda incluirse en el tipo 4 de Kierner, al igual que el informe de Arun et al., motivo por el cual esa clasificación no fue utilizada. Otras clasificaciones, como la propuesta por Wang et al. nos parece dificil de aplicar en la práctica.

En nuestros hallazgos, si consideramos la clasificación de Cernea, encontramos en el lado izquierdo un $52 \%$ de nervios en riesgo y en el lado derecho un $44 \%$. En cambio si consideramos la clasificación de Friedman, según nuestros hallazgos el $56 \%$ de los RLNLS izquierdos están dentro del grupo de riesgo, mientras que el $60 \%$ lo están en el lado derecho, al pertenecer estos al tipo 1.

Según Cernea et al., el $37 \%$ estan en este grupo de alto riesgo, mientras que para Kierner et al., este porcentaje aumenta al $58 \%$ (9\%). Nuestro reporte informa un porcentaje mayor de nervios en riesgo, aunque según Estrela et al. que informan un $68 \%$ pertenecientes a este grupo, ellos sostienen que el nervio siempre está en riesgo por la proximidad de las estructuras ligadas y seccionadas o resecadas en la cirugía tiroidea (Estrela et al.).

Para Ekhar et al., los nervios en riesgo se presentaron en el $65 \%$, con una mayor prevalencia del tipo $2 \mathrm{~b}$ en el lado izquierdo, similar a nuestros hallazgos. Arun et al. re- 
fieren que el $67 \%$ pertenecen al grupo de riesgo, mientras que para Aina \& Hisham este porcentaje es del $83 \%$, similar hallazgo al reportado por Pagedar \& Freeman.

La ATS es una de las arterias más importantes del cuello, nace habitualmente como primera rama de la ACE o bien a nivel de la bifurcación carotidea. El origen de esa arteria está asociada no solo con su patrón de ramificación sino también con el nivel o altura de la $\mathrm{BC}$, puesto que en aquellos casos en que nace de la carótida común, la BC está en una posición más alta, por encima del margen superior del cartílago tiroideo (Dessie).

En este trabajo, la ATS nació de la ACE en el $64 \%$ de los casos, mientras que de la $\mathrm{BC}$ en el $36 \%$. Este patrón se repitió en ambos lados, aunque la simetríase dió en 18 casos (72\%). Dessie reporta a la ACE como origen de la ATS en el $45 \%$, mientras que a la BC en el $28 \%$. El porcentaje restante nace de la arteria carótida común (ACC). No analiza la asociación entre el sitio de nacimiento de la ATS y el punto de entrecruzamiento con el nervio en cuestión. Nosotros no encontramos nacimientos en este último nivel. Sreedharan et al. (2018) en 30 cadáveres econtraron que el $88 \%$ de las ATS nacía de la ACE, mientras que el $8 \%$ lo hacía de la BC y el $4 \%$ de la ACC. En este trabajo se relata que en dos casos en que la ATS nacía a nivel de la ACC no se presentaron entrecruzamientos con el RLNLS (Sreedharan et al.). Patel et al. (2013) en 50 cadáveres, presentan una casuística parecida a la nuestra con el $77 \%$ de nacimientos a nivel de la ACE, el $23 \%$ en la BC y sin ninguna arteria emergiendo de la ACC (Patel et al.). Quijano Blanco \& Luque Bernal (2015) refieren que en 67 cadáveres colombianos el $100 \%$ se originó en la ACE.

Won (2016) en un estudio con 30 cadáveres encontró que el nacimiento de la ATS se daba mayoritariamente a nivel del tronco carotídeo, $83 \%$, tanto de la ACE como de la BC o de la ACC, sin embargo encontró que el $17 \%$ puede nacer del tronco tirolingual o linguafacial. En base a esto presentó una clasificación con tres tipso; tipo 1 nacimiento a nivel de la ACE, tipo 2 a nivel de la BC y tipo 3 en la ACC, $1 \mathrm{~cm}$ por debajo de la bifurcación. No analiza la asociación entre estos tipos y el nivel de entrecruzamiento vasculo nervioso estudiado en este trabajo (Quijano Blanco \& Luque Bernal).

En la casuística presentada en este trabajo, se encontró asociación con significación estadística entre el punto de origen de la arteria y los tipos de Cernea en el lado izquierdo. También encontramos asociación entre la simetríade este nacimiento y los tipos de Cernea, pero en este punto sin significación estadística.
La clasificación de Friedman se basa en el sitio en que el RLNLS perfora al músculo constrictor inferior de la faringe para posteriormente alcanzar al MCT. En el tipo 1, el nervio transcurre superficial hasta el músculo constrictor inferior de la faringe y este tipo es considerado de alto riesgo mientras que el tipo 3, muchas veces no es identificable, perfora al músculo constrictor inferior de la faringe en su parte alta, siendo este tipo menos expuesto a lesiones. Este tipo se presenta en el $20 \%$ de los casos aproximadamente (Ekhar et al.). Como puede verse en la Tabla I las variantes peligrosas se presentaron en este trabajo en el $56 \%$ en el lado izquierdo y en el $60 \%$ en el lado derecho, mientras que el tipo 3 se presentó en el $24 \%$ y el $16 \%$ respectivamente. Lo cual concuerda con lo informado en la literatura.

Los tipos con "nervios en riesgo" de la clasificacion de Cernea (tipo 2) y de Friedman (tipo 1) no presentaron asociación en este trabajo

\section{CONCLUSIONES}

Según el nivel de entrecruzamiento entre el RLNLS y la ATS con respecto al PSGT, el porcentaje de "nervios en riesgo" en este trabajo fue de $52 \%$ para el lado izquierdo y $44 \%$ para el lado derecho. Se encontró una asociación positiva, con significación estadística, entre el nivel de origen de la ATS a nivel del BC y el tipo 2 de Cernea para el lado izquierdo

El tipo 1 de Friedman se presentó en el $56 \%$ del lado izquierdo y en el $60 \%$ en el lado derecho, mientras que la perforación alta del músculo constrictor inferior de la faringe se presentó en el $24 \%$ y $16 \%$, respectivamente, considerándose a los del primer grupo de alto riesgo y a los de este último como nervios protegidos por la cobertura del músculo constrictor inferior de la faringe.

No se encontró asociación entre las clasificaciones de Cernea y Friedman con respecto a los hallazgos de "nervios en riesgo".

MEDINA-RUIZ, B. A.; MEDINA-IZCURDIA, J. J.; MEDINAIZCURDIA, B. A.; MARTÍNEZ-VERA, R. B.; MARTÍNEZVERA, P. R.; IZCURDIA, C. E. \& OTTONE, N. E.Topographic anatomy of the upper laryngeal nerve: Surgical importance in thyroidectomies. Int. J. Morphol., 38(4):766-773, 2020.

SUMMARY: In surgeries on the thyroid gland, much attention has been given to the management of the inferior laryngeal nerve and parathyroid glands, but not the external branch of the 
given by the aforementioned nerve and the superior thyroid artery. This paper intends to study these relationships based on the meticulous dissection of the larynx-sternothyroid triangle ("Joll triangle") of 25 formolized adult corpses. As a relevant finding, it is reported that the "nerves at risk" according to the Cernea classification, which is based on the distance less than one centimeter at the intersection of the nerve with the superior thyroid artery with respect to the upper pole of the gland, is $52 \%$ for the right side and $44 \%$ for the left side of the neck. The low origin of the artery at the level of the carotid bifurcation is associated with a greater number of "nerves at risk" on the left side. According to the penetration point of the external branch of the superior laryngeal nerve in the inferior pharyngeal constrictor muscle, the Friedman classification is established, very useful especially in surgeries aided by neurostimulation. In this classification the "nerves at risk" are those that run superficially to the muscle, while the protected nerves would be those that pierce the muscle in its upper part. In tis work, the "nerves at risk" presented on the left side in $56 \%$ of the cases and the right side in $60 \%$, while those "protected" in $24 \%$ and 16 $\%$ respectively.

KEY WORDS: External branch of the superior laryngeal nerve; Superior thyroid artery; Superior pole of the thyroid gland; Topographic anatomy.

\section{REFERENCIAS BIBLIOGRÁFICAS}

Aina, E. N. \& Hisham, A. N. External laryngeal nerve in thyroid surgery: recognition and surgical implications. A. N. Z. J. Surg., 71(4):212-4, 2001.

Arun, S.; Sreejayan, M. P. \& Anoop, N. Title: Topographical anatomy of external branch of superior laryngeal nerve as related to thyroidectomy. J. Med. Sci. Clin. Res., 4(11):13885-90, 2016.

Bruno, O. D. Why did Amelita Galli-Curci lose her voice? Arch. Endocrinol. Metab., 63(4):314-7, 2019.

Cernea, C. R.; Ferraz, A. R.; Nishio, S.; Dutra, A. Jr.; Hojaij, F. C. \& dos Santos, L. R. Surgical anatomy of the external branch of the superior laryngeal nerve. Head Neck, 14(5):380-3, 1992.

Dessie, M. A. Variations of the origin of superior thyroid artery and its relationship with the external branch of superior laryngeal nerve. $P L O S$ One, 13(5): $\mathrm{e} 0197075,2018$.

Ekhar, V. R.; Ramkumar, V.; Shelkar, R. N. \& Sarode, A. V. Identification of external branch of superior laryngeal nerve during thyroid surgery: a prospective study. Int. J. Otorhinolaryngol. Head Neck Surg., 4(1):228-32, 2018.

Estrela, F.; Leão, H. Z. \& Jotz, G. P. Anatomic relation between the external branch of the superior laryngeal nerve and the thyroid gland. Braz. J. Otorhinolaryngol., 77(2):249-58, 2011.

Friedman, M.; LoSavio, P. \& Ibrahim, H. Superior laryngeal nerve identification and preservation in thyroidectomy. Arch. Otolaryngol. Head Neck Surg., 128(3):296-303, 2002.

Gurleyik, E. Motor interconnections between superior and inferior laryngeal nerves. Cereus, 10(3):e2337, 2018.

Gurleyik, E.; Dogan, S.; Cetin, F. \& Gurleyik, G. Visual and electrophysiological identification of the external branch of superior laryngeal nerve in redo thyroid surgery compared with primary thyroid surgery. Ann. Surg. Treat. Res., 96(6):269-74, 2019.

Ha, E. J.; Baek, J. H. \& Lee, J. H. Ultrasonography-based thyroidal and perithyroidal anatomy and its clinical significance. Korean J. Radiol., 16(4):749-66, 2015.
Kandil, E.; Mohamed, S. E.; Deniwar, A.; Mohamed, H.; Friedlander, P.; Aslam, R.; Saeed, A.; Musa, I. \& Randolph, G. Electrophysiologic identification and monitoring of the external branch of superior laryngeal nerve during thyroidectomy. Laryngoscope, 125(8):19962000, 2015.

Kierner, A. C.; Aigner, M. \& Burian, M. The external branch of the superior laryngeal nerve: its topographical anatomy as related to surgery of the neck. Arch. Otolaryngol. Head Neck Surg., 124(3):301-3, 1998.

Manzini, J. L. Declaración De Helsinki: principios éticos para la investigación médica sobre sujetos humanos. Acta Bioethica, 6(2):323-34, 2000.

Maranillo, E.; León, X.; Quer, M.; Orús, C. \& Sañudo, J. R. Is the external laryngeal nerve an exclusively motor nerve? The cricothyroid connection branch. Laryngoscope, 113(3):525-9, 2003.

Menon, R. R.; Murali, S.; Nair, C. G.; Babu, M. J. C. \& Jacob, P. Correlation between the Cernea Classification of External Branch of Superior Laryngeal Nerve in Relation to the Ultrasound-based Volume of Thyroid Gland. Indian J. Endocrinol. Metab., 21(6):845-7, 2017.

Mizrachi, A.; Swartzwelder, C. E. \& Shaha, A. R. Proposal for anatomical classification of the superior pole in thyroid surgery. J. Surg. Oncol., 112(1):15-7, 2015.

Pagedar, N. A. \& Freeman, J. L. Identification of the external branch of the superior laryngeal nerve during thyroidectomy. Arch. Otolaryngol. Head Neck Surg., 135(4):360-2, 2009.

Patel, J. P.; Dave, R. V.; Shah, R. K.; Kanani, S. D. \& Nirvan, A. B. A study of superior thyroid artery in 50 cadavers. Int. J. Biol. Med. Res., 4(1):2875-8, 2013.

Quijano Blanco, Y. \& Luque Bernal, R. M. Thyroid gland irrigation in a sample of colombian population. Int. J. Morphol., 33(3):1032-7, 2015.

Sanders, I.; Wu, B. L.; Mu, L.; Li, Y. \& Biller, H. F. The innervation of the human larynx. Arch. Otolaryngol. Head Neck Surg., 119(9):934-9, 1993.

Sreedharan, R.; Krishna, L. \& Shetty, A. Origin of superior thyroid artery: under the surgeon's knife. J. Vasc. Bras., 17(4):290-5, 2018.

Testut, L. \& Latarjet, A. Tratado de Anatomía Humana. Tomo III. Barcelona, Salvat Editores, 1984. pp.168-9.

Wang, K.; Cai, H.; Kong, D.; Cui, Q.; Zhang, D. \& Wu, G. The identification, preservation and classification of the external branch of the superior laryngeal nerve in thyroidectomy. World J. Surg., 41(10):2521-9, 2017.

Whitfield, P.; Morton, R. P. \& Al-Ali, S. Surgical anatomy of the external branch of the superior laryngeal nerve. A. N. Z. J. Surg., 80(11):813-6, 2010.

Dirección para Correspondencia:

Prof. Dr. Blas. A. Medina Ruiz

Profesor Adjunto de Anatomía descriptiva y Topográfica

Facultad de Ciencias Médicas

Universidad Nacional de Asunción (FCM-UNA)

Asunción

PARAGUAY

Email: bamci@hotmail.com

Recibido: 29-12-2019

Aceptado:17-01-2020 